\title{
High Code Rate Low-Density Parity-Check Codes for Optical Communication Systems
}

\author{
Ivan B. Djordjevic and Bane Vasic, Senior Member, IEEE
}

\begin{abstract}
A novel family of low-density parity-check codes based on symmetrically repeated difference systems is proposed. Codes from this family have very high code rate, girth at least six, large minimum distance, and significantly outperform conventional Reed-Solomon codes.
\end{abstract}

Index Terms-Forward error correction (FEC), low-density parity-check codes, optical communications, symmetrically repeated difference systems (SRDSs).

\section{INTRODUCTION}

$\mathbf{T}$ HE ROLE of forward error correction (FEC) in maintaining acceptable transmission quality is becoming increasingly important as the capacity of dense wavelengthdivision-multiplexing (WDM) long-haul transmission systems grows. In state-of-the-art optical communication systems, two FEC standards are currently in use, both based on BoseChaudhuri-Hocquenghen (BCH) codes [1]. For example, "in-band" FEC used in synchronous optical network/synchronous digital heirarchy (SONET/SDH) framing is a 3-[4359,4320] shortened BCH code (ITU-T G.707 standard). "Out-band" FEC coding is accomplished by using (interleaved) Reed-Solomon (RS) [255, 238] codes, as specified in the digital wrapper of the ITU G.709 standard. The FEC used in submarine systems is based on an RS with parameters [255, 239], as specified by the ITU-T G.975 standard [2]. For optical communication systems with a bit rates beyond $10 \mathrm{~Gb} / \mathrm{s}$, more powerful FEC codes with larger coding gains and high code rates are desirable. Two such schemes have been proposed recently: Ait Sab et al. [3] proposed a concatenated scheme with two RS codes, and Pyndiah [4] and Ait Sab and Lemarie [5] proposed block turbo codes.

Recently, we showed in [6]-[8] that the error performance and hardware complexity of turbo codes can be outperformed by low-density parity check (LDPC) codes. In [7], the projective geometry-based [1 057 813] LDPC code of minimum distance 34 with net coding gain of $10.5 \mathrm{~dB}$ at bit-error rate (BER) below $10^{-10}$ is proposed, outperforming the block turbo code of the minimum distance 30 presented in [17] for $0.4 \mathrm{~dB}$ in coding gain. Moreover, the LDPC code based on the projective

\footnotetext{
Manuscript received September 12, 2003; revised February 10, 2004. This work was supported by the National Science Foundation (NSF) under Grants CCR 0208597 and ITR 0325979.

I. B. Djordjevic is with the Department of Electrical and Computer Engineering, University of Arizona, Tucson, AZ 85721 USA, on leave from the Faculty of Computing, Engineering and Mathematical Sciences, University of the West of England, Bristol BS16 1QY, U.K. (e-mail: ivan@ece.arizona.edu).

B. Vasic is with the Department of Electrical and Computer Engineering, University of Arizona, Tucson, AZ 85721 USA (e-mail: vasic@ece.arizona.edu).

Digital Object Identifier 10.1109/LPT.2004.826807
}

geometry $\mathrm{PG}(2,64)$ (from the same family) has the minimum distance 66 and would perform even better. LDPC codes can be designed in a pseudorandom fashion [9], but this leads to encoders-decoders with complexity exceeding practical limits imposed on high-speed optical communication systems. A more attractive approach that we propose in this letter is to design block-cyclic LDPC codes based on a new class of combinatorial objects that allow encoding and decoding to be performed via fast and simple circuits. Such high-speed FEC architectures are of crucial importance in optical communications, and there has been a great deal of activity in this area. For example, Agere Systems (see Azadet et al. [10]) reported an optical networking interface device with four parallel RS codecs, each operating at $2.5 \mathrm{~Gb} / \mathrm{s}$. Agere Systems [11], [12] has also implemented a rate $1 / 2$ block length 1024 LDPC code for optical networking, and Flarion [13] researchers developed codecs employing proprietary multiedge type LDPC code of length 4096.

In this letter, a novel class of LDPC codes based on symmetrically repeated difference systems (SRDSs) is proposed. High code rate, large minimum distance, girth of at least six, low complexity encoding and decoding, and excellent BER performance make these codes strong candidates for high-speed applications such as optical communications and networks. The performance of the proposed codes is assessed in a very realistic simulation environment (for more details, see [7], [15]) that includes impairments originating from amplified spontaneous emission (ASE) noise, pulse distortions due to fiber nonlinearities, chromatic dispersion, crosstalk effects, and intersymbol interference. Since the coding gain of these LDPC codes is significantly higher than the gain of RS codes of larger redundancy, they are excellent candidates for use in metropolitan area network (MAN) and wide area network (WAN) applications, and long-haul transmission as well.

\section{CODE DESCRIPTION}

The codes proposed in this letter are based on the theory of difference systems [14], more precisely on the SRDSs also known as mixed difference systems.

The sets $S_{1}, S_{2}, \ldots, S_{t}$ of size $k$ are said to form a $(v, k, \lambda)$ difference system in an additive abelian group $G$ of order $v$ if the differences arising from $S_{i}$ gives each nonzero element of $G$ at most $\lambda$ times. If we further allow taking several copies of each element distinguished by a subscript, we are able to construct much more powerful designs called here SRDSs or mixed difference systems. For example, let $G=Z_{3}$ and $t=3$, and observe the blocks: $\left\{1_{1}, 2_{1}, 0_{2}\right\},\left\{1_{2}, 2_{2}, 0_{3}\right\},\left\{1_{3}, 2_{3}, 0_{1}\right\}$, and $\left\{0_{1}, 0_{2}, 0_{3}\right\}$. 
The differences arising from elements with the same subscript are called pure differences, and those arising from elements with different subscripts are called mixed differences. The $(1,1)$ pure differences are $1-2=2,2-1=1$, i.e. all nonzero elements of $Z_{3}$. The $(2,2)$ pure differences are $1-2=2,2-1=1 ;(2,3)$ mixed differences are $2-0=2,1-0=1,0-0=0$, etc. Therefore, every nonzero element from $Z_{3}$ can be represented as difference of two elements with the same or different subscript exactly once, and four blocks above form an SRDS (mixed difference system). By cyclically shifting each of the block from initial set $(\bmod 3)$ twice, giving subscripts unchanged, the following 12 blocks form $(9,3,1)$ balanced incomplete block design (BIBD) or Steiner triple system of order nine:

$$
\begin{aligned}
& \left\{1_{1}, 2_{1}, 0_{2}\right\},\left\{1_{2}, 2_{2}, 0_{3}\right\},\left\{1_{3}, 2_{3}, 0_{1}\right\},\left\{0_{1}, 0_{2}, 0_{3}\right\} \\
& \left\{2_{1}, 0_{1}, 1_{2}\right\},\left\{2_{2}, 0_{2}, 1_{3}\right\},\left\{2_{3}, 0_{3}, 1_{1}\right\},\left\{1_{1}, 1_{2}, 1_{3}\right\} \\
& \left\{0_{1}, 1_{1}, 2_{2}\right\},\left\{0_{2}, 1_{2}, 2_{3}\right\},\left\{0_{3}, 1_{3}, 2_{1}\right\},\left\{2_{1}, 2_{2}, 2_{3}\right\} .
\end{aligned}
$$

For more details on BIBDs and Steiner triple systems and their use in a design of the LDPC codes, please refer to [8], [14].

The following construction method due to Bose [16, p. 80] provide a very powerful method of designing a number of different BIBDs.

Let $S$ be a set of $m$ elements. Associate to each element $u$ from $S n$ symbols $u_{1}, u_{2}, \ldots, u_{n}$. Let sets $S_{1}, S_{2}, \ldots, S_{t}$ satisfy the following conditions: 1$)$ every set $S_{i}(i=1, \ldots, t)$ contains $k$ symbols (the symbols from the same set are different from one another); 2) among $k t$ symbols in $t$ sets exactly $r$ symbols belong to each of $n$ classes $(n r=k t)$; and 3) the differences from $t$ sets are symmetrically repeated so that each repeats $\lambda$ times. If $s$ is an element from $S$, from each $S_{i}$ set we are able to form another set $S_{i, s}$ by adding $s$ to $S_{i}$ keeping the class number (subscript) unchanged; then $m t$ sets $S_{i, s}(i=1, \ldots, t ; s \in S)$ represent a $(m n, n t, r, k, \lambda)$ BIBD.

Example: The following blocks $\left\{1_{i},(2 t)_{i}, 0_{i+1}\right\},\left\{2_{i},(2 t-\right.$ $\left.1)_{i}, 0_{i+1}\right\}, \ldots,\left\{t_{i},(t+1)_{i}, 0_{i+1}\right\}(i \in\{1,2,3\}),\left\{0_{1}, 0_{2}, 0_{3}\right\}$, where the elements are observed per $\bmod 2 t+1$, and subscripts per mod 3 , from a $(6 t+3,3,1)$ SRDS or Steiner triple system of order $6 t+3(t \geq 0)$. By considering the elements of the blocks as position of ones within the corresponding columns of an element-block incident matrix, the matrix of parity-checks can be generated. Since overlapping between any two columns (or rows) is at most one the LDPC code of girth at least six is obtained.

For $t=1$, the following matrix of parity-check is given as an illustration

$$
H=\left[\begin{array}{llllllllll}
1 & 0 & 1 & 1 & 0 & 0 & 0 & 1 & 0 & 0 \\
0 & 1 & 0 & 1 & 0 & 0 & 0 & 0 & 1 & 0 \\
0 & 1 & 1 & 0 & 0 & 0 & 0 & 0 & 0 & 1 \\
1 & 1 & 0 & 0 & 0 & 1 & 1 & 0 & 0 & 0 \\
0 & 0 & 1 & 0 & 1 & 0 & 1 & 0 & 0 & 0 \\
0 & 0 & 0 & 1 & 1 & 1 & 0 & 0 & 0 & 0 \\
1 & 0 & 0 & 0 & 1 & 0 & 0 & 0 & 1 & 1 \\
0 & 0 & 0 & 0 & 0 & 1 & 0 & 1 & 0 & 1 \\
0 & 0 & 0 & 0 & 0 & 0 & 1 & 1 & 1 & 0
\end{array}\right] .
$$

The code rate can be estimated as $R \cong 1-3 /(3 t+1)$, the code length as $b \cong(2 t+1)(3 t+1)$, and the number of parity bits as $6 t+3$. Therefore, for code lengths larger than 500 , the code rate is $\geq 0.9$, and the construction is suitable for high

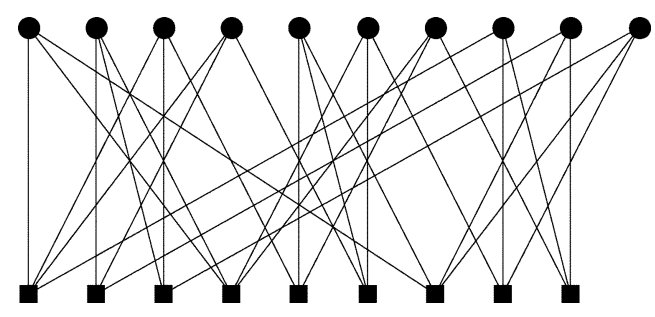

Fig. 1. Bipartite graph for parity-check matrix from Section II.

code rate applications. It will be shown in Section IV that LDPC codes constructed in this way have excellent BER performance in optical communication channel.

\section{Min-Sum DeCODING AlgORITHM}

The codes proposed in this letter have the girth six and can be decoded by various decoding methods such as one-step majority-logic, bit-flipping, and iterative decoding based on the sum-product (message-passing) algorithm. Although the sumproduct iterative decoding has been demonstrated to perform well in various types of channels, it is computationally extensive and it is not clear if it is suitable for optical communications at data rate 10 or $40 \mathrm{~Gb} / \mathrm{s}$ or higher. However, the min-sum algorithm, which is an approximation of the a posteriory probability decoding, requires only simple addition and finding minimum operations and, as such, is suitable for high-speed optical transmission, and it is implemented in this letter.

For any codeword $x=\left(x_{v}\right)_{1<v<n}$ in a linear block code given by the parity check matrix $H$, the following set of equations is satisfied:

$$
\sum_{v} h_{c, v} x_{v}=0, \quad 1 \leq c \leq n-k
$$

known as parity check equations. Iterative decoding can be visualized as message passing on a bipartite graph representation, called Tanner graph, of the parity check matrix [8]. There are two types of vertices in the graph: check vertices (check nodes) indexed by $c$ and variable vertices (bit nodes) indexed by $v$. An edge connecting vertices $c$ and $v$ exists if $h_{c, v}=1$, i.e., if variable $v$ participates in the parity check equation $c$. For example, the bipartite graph corresponding to the parity check matrix from Section II is shown in Fig. 1.

Decoding can be done as follows. First, a priori information of the bit at position $v, \mu_{v}^{(0)}$ is taken as the channel sample and messages passed from node $v$ to node $c$ in the bipartite graph $\lambda_{v, c}^{(0)}$ are initialized to $\mu_{v}^{(0)}$.

In the $j$ th iteration, we update the messages to be passed from check node $c$ to bit node $v, \Lambda_{c, v}^{(j)}$, as

$$
\Lambda_{c, v}^{(j)}=\prod_{w \neq v} \operatorname{sign}\left(\lambda_{w, c}^{(j-1)}\right) \cdot \min _{w \neq v}\left|\lambda_{w, c}^{(j-1)}\right|
$$

and messages to be passed from bit node $v$ to check node $c, \lambda_{v, c}^{(j)}$, according to

$$
\lambda_{v, c}^{(j)}=\mu_{v}^{(0)}+\sum_{d \neq c} \Lambda_{d, v}^{(j)}
$$




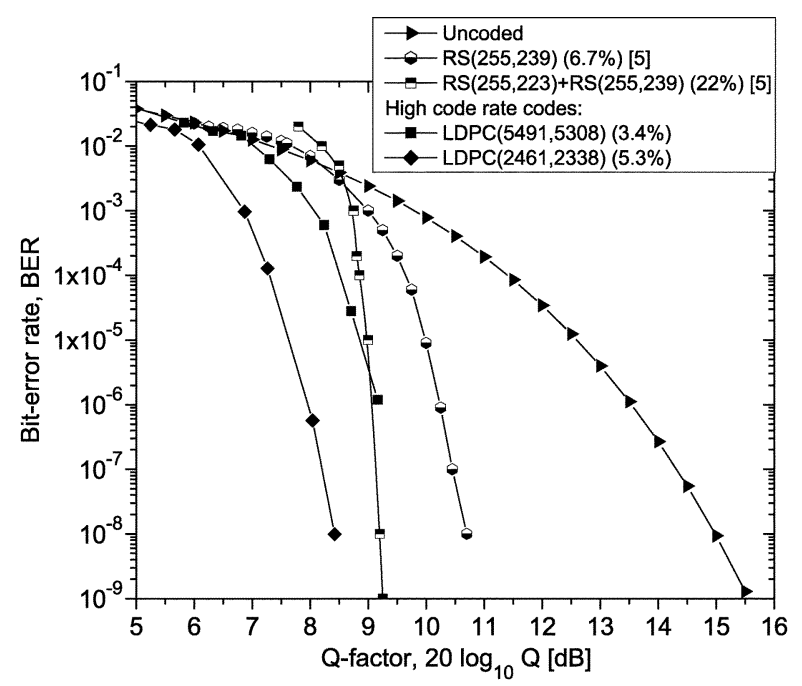

Fig. 2. BER performance of symmetrically repeated differences based LDPC codes at $40 \mathrm{~Gb} / \mathrm{s}$ with six iterations in min-sum algorithm.

The last step in iteration $j$ is to compute updated normalized log-likelihood ratios $\mu_{v}^{(j)}$ according to

$$
\mu_{v}^{(j)}=\mu_{v}^{(0)}+\sum_{c} \Lambda_{c, v}^{(j)} .
$$

For each bit $x_{v}$ the estimation is made according to

$$
\hat{x}_{v}=\left\{\begin{array}{ll}
1, & \text { if } \mu_{v}^{(j)}<0 \\
0, & \text { otherwise }
\end{array} .\right.
$$

Decoding halts when a valid codeword $\left(\sum_{v} h_{c, v} \hat{x}_{v}=0\right)$ or a maximum number of iteration has been reached. The steps (2) and (3) can be viewed as propagation of "beliefs" in a code bipartite graph [8].

\section{Performance Analysis}

Fig. 2 plots the BER obtained from Monte Carlo simulations with six iterations for a symmetric repeated differences BIBD-based LDPC [5491,5308] code with code rate $R=0.967$ (redundancy of 3.4\%), and an SRDS-based LDPC [2461,2338] with code rate 0.95 (redundancy of 5.3\%). A WDM system with $40-\mathrm{Gb} / \mathrm{s}$ bit rate per channel and a channel spacing of $100 \mathrm{GHz}$ is considered. It is assumed that the observed channel is located at $1552.524 \mathrm{~nm}(193.1 \mathrm{THz})$ and that there exists a nonnegligible interaction with six neighboring channels. The influence of optical and electrical filters is taken into account as well. The transmission system considered has a dispersion map composed of a single-mode fiber (SMF) section (of length $80 \mathrm{~km}$ ), followed by an erbium-doped fiber amplifier (EDFA) to compensate for the fiber losses in the SMF section, and a dispersion compensating fiber (DCF) section to compensate both for group-velocity dispersion (GVD) and second-order GVD, as well as another EDFA to compensate for the fiber losses in the DCF section. Eight SMF-DCF sections are considered and the $Q$ factor is additionally decreased by noise loading. Although of much smaller redundancy $(5.3 \%)$, the LDPC [2461,2338] performs much better than the conventional $\mathrm{RS}(255223)+\mathrm{RS}(255239)$ concatenation scheme (of redundancy $22 \%$ ). Notice that the complexity of the min-sum algorithm is significantly lower than the complexity of the RS decoding algorithm, since the only operations performed are addition and calculating the minimum. The comparison with RS codes is not completely fair because Fig. 2 does not show LDPC BERs at $10^{-13}$ or $10^{-15}$. Based on previous results [7], [8] as well as recent results by Richardson [18], we believe that the proposed code would not experience the error floor. The existence of a BER floor of LDPC codes is still an open issue, although there has been some progress recently [18].

\section{CONCLUSION}

We have proposed a novel class of FEC regular LDPC codes with very high code rates $(\geq 0.9)$, girth at least six, and large minimum distance, based on SRDSs.

The proposed LPDC codes perform well in the presence of ASE noise, fiber nonlinearities, chromatic dispersion, and intersymbol interference, significantly outperform traditional FEC schemes based on RS codes (of higher redundancy), and are suitable for MAN and WAN applications as well as for highspeed long-haul optical transmission systems.

\section{REFERENCES}

[1] F. J. MacWilliams and N. J. A. Sloane, The Theory of Error-Correcting Codes. Amsterdam, The Netherlands: Elsevier, 1977.

[2] Forward Error Correction for Submarine Systems, ITU Standard Tech. Recommendation G.975/G709.

[3] O. A.O. Ait Sab et al., "Concatenated forward error correction schemes for long-haul DWDM optical transmission systems," in Proc. Eur. Conf. Optical Communication (ECOC), 1999, p. II-290.

[4] R. M. Pyndiah, "Near optimum decoding of product codes: Block turbo codes," IEEE Trans. Commun., vol. 46, pp. 1003-1010, Aug. 1998.

[5] O. A. Sab and V. Lemarie, "Block turbo code performances for long-haul DWDM optical transmission systems," in Proc. Optical Fiber Communication (OFC), vol. 3, 2001, pp. 280-282.

[6] B. Vasic and I. B. Djordjevic, "Low-density parity check codes for long haul optical communications systems," IEEE Photon. Technol. Lett., vol. 14, pp. 1208-1210, Aug. 2002.

[7] I. B. Djordjevic and B. Vasic, "Projective geometry LDPC codes for ultralong-haul WDM high-speed transmission," IEEE Photon. Technol. Lett., vol. 15, pp. 784-786, May 2003.

[8] B. Vasic, I. B. Djordjevic, and R. Kostuk, "Low-density parity check codes and iterative decoding for long haul optical communication systems," J. Lightwave Technol., vol. 21, pp. 438-446, Feb. 2003.

[9] D. J. C. MacKay, "Good error correcting codes based on very sparse matrices," IEEE Trans. Inform. Theory, vol. 45, pp. 399-431, Mar. 1999.

[10] K. Azadet et al., "Equalization and FEC techniques for optical transceivers," IEEE J. Solid-State Circuits, vol. 37, pp. 317-327, Mar. 2002.

[11] C. Howland and A. Blanksby, "A $220 \mathrm{~mW} 1 \mathrm{~Gb} / \mathrm{s}$ 1024-bit rate-1/2 low density parity check code decoder," in IEEE Conf. Custom Integrated Circuits, 2001, pp. 293-296.

[12] — , "Parallel decoding architectures for low density parity check codes," in Proc. ISCAS 2001, vol. 4, 2001, pp. 742-745.

[13] T. Richardson and R. Urbanke, "The renaissance of Gallager's low-density parity-check codes," IEEE Commun. Mag., vol. 41, pp. 126-131, Aug. 2003.

[14] I. Anderson, Combinatorial Designs: Construction Methods. Chichester, U.K.: Ellis Horwood Ltd., 1990.

[15] I. B. Djordjevic and B. Vasic, "An advanced direct detection receiver model," J. Optical Commun., vol. 25, no. 1, pp. 6-9, Feb. 2004.

[16] D. Raghavarao, Constructions and Combinatorial Problems in Design of Experiments. New York: Dover, 1988.

[17] T. Mizuochi et al., "Experimental demonstration of net coding gain of $10.1 \mathrm{~dB}$ using $12.4 \mathrm{~Gb} / \mathrm{s}$ block turbo code with 3-bit soft decision," in Proc. Optical Fiber Communication (OFC 2003), 2003, Postdeadline Papers, pp. PD21-1-PD21-3.

[18] T. Richardson, "Error floors of LDPC codes," in Proc. 41st Allerton Conf. Communications, Control and Computing, Oct. 2003. 